Case Report

\title{
A case of focal segmental glomerulosclerosis in a young girl with a very low birth weight
}

\author{
Yasuyo Kashiwagi ${ }^{1}$, Kazushi Agata ${ }^{1}$, Gaku Yamanaka ${ }^{1}$ and Hisashi Kawashima ${ }^{1 *}$
}

\author{
Department of Pediatrics and Adolescent Medicine, Tokyo Medical University, 6-7-1 Nishishinjuku, Shinjuku- \\ ku, Tokyo 160-0023, Japan; whatwouldjesusdo721@yahoo.co.jp (K.A.); gaku@tokyo-med.ac.jp (G.Y); \\ hisashi@tokyo-med.ac.jp (H.K.) \\ Corresponding author: Yasuyo Kashiwagi, M.D., Ph.D. \\ Department of Pediatrics and Adolescent Medicine \\ Tokyo Medical University \\ 6-7-1 Nishishinjuku, Shinjuku-ku, Tokyo, Japan \\ Tel.: +81(0)3-3342-6111; Fax: +81(0)3-3344-0643 \\ E-mail: hoyohoyo@tokyo-med.ac.jp
}

\begin{abstract}
In Japan, the prevalence of low birth weight (LBW) has been estimated to be approximately $10 \%$, which is the highest among developed countries. This high prevalence might affect the prevalence of LBW-associated diseases in the adult population of Japan. Recently, LBW has been recognized as a contributing factor to post-adaptive focal segmental glomerulosclerosis (FSGS) in adulthood; however, few reports to date have evaluated the clinical and pathological characteristics of post-adaptive FSGS. A 13-year-old girl was referred to our hospital owing to mild proteinuria, which was detected at a school urinary screening. She was born at a gestational age of 23 weeks, with a very LBW of $630 \mathrm{~g}$. Dipstick urinalysis revealed grade $(2+)$ proteinuria. Her serum creatinine level was $1.02 \mathrm{mg} / \mathrm{dL}$, and she was diagnosed as having stage 2 chronic kidney disease (CKD). Her serum uric acid level was $7 \mathrm{mg} / \mathrm{dL}$. Her mother and 16-years old brother had hyperuricemia, too. A percutaneous renal biopsy leads to a diagnosis of FSGS. After 3 years of treatment with an angiotensin receptor blocker, her proteinuria decreased. However, her serum creatinine level was 1.07 $\mathrm{mg} / \mathrm{dL}$, and she still had stage $2 \mathrm{CKD}$. We considered that in this patient, the first hit was her LBW, and the second hit was hyperuricemia. The second hit might be associated with the development of CKD. The birth history of patients is not usually confirmed by nephrologists. Our case demonstrates that obtaining information regarding the preterm birth and LBW of patients is important in the diagnosis of noncommunicable diseases, because school urinary screening is not routinely performed in countries other than Japan.
\end{abstract}

Keywords: chronic kidney disease; low birth weight; focal segmental glomerulosclerosis; two-hit theory

\section{Introduction}

Preterm birth rates have increased recently worldwide, and about 13 million infants are born preterm each year [1]. Clinical and experimental lines of evidence have demonstrated that preterm and low birth weight (LBW) infants are at risk of hypertension, and cardiovascular and renal dysfunction in adulthood [2]. This is the concept of the developmental origins of health and disease, which play a potential role in determining the susceptibility to developing noncommunicable diseases (such as cardiovascular disease and chronic kidney disease (CKD) in adulthood [3]. In Japan, the prevalence of LBW has been estimated to be approximately $10 \%$, which is the highest in developed countries. Therefore, this high prevalence might affect the prevalence of LBW-associated diseases in the adult population of Japan [4]. Recently, LBW has been recognized as a contributing factor to the development of post-adaptive focal segmental glomerulosclerosis (FSGS) in 
adulthood [5]. However, few studies to date have evaluated the clinical and pathological characteristics of post-adaptive FSGS.

\section{Case Report}

A 13-year-old girl was referred to our hospital owing to mild proteinuria, which was detected at a school urinary screening. No urinary abnormalities had been detected previously. She was born at a gestational age of 23 weeks, with a very LBW of $630 \mathrm{~g}$, and had chronic lung disease and premature infant retinitis. She required $\mathrm{O}_{2}$ until 1 year of age; however, her growth and development had reached the normal range by that time. Apart from retinopathy of prematurity, she had been generally healthy.

Physical examination showed the following: height $154 \mathrm{~cm}$, weight $50 \mathrm{~kg}$, body mass index 21.1, and blood pressure 115/73 mmHg. Dipstick urinalysis revealed grade (2+) proteinuria. Her serum creatinine level was $1.02 \mathrm{mg} / \mathrm{dL}$, and she was diagnosed as having stage 2 CKD based on reference serum creatinine levels of Japanese male and female children aged 12 to 15 years. Her serum uric acid level was $7 \mathrm{mg} / \mathrm{dL}$ (normal level was less than $4.6 \mathrm{mg} / \mathrm{dL}$ based on reference serum levels of female children aged 12 to 14 years), she had hyperuricemia [6]. Her mother and her 16-years old brother had hyperuricemia, too.

A percutaneous renal biopsy demonstrated that 1 glomerulus had segmental sclerosis with adhesion to the Bowman's capsule, and partial focal interstitial fibrosis was also observed (Figure 1). The mean diameter of the glomeruli was $348.23 \mu \mathrm{m}$, which was much larger than that of normal glomeruli $(168 \pm 12 \mu \mathrm{m})$ [7]. Immunofluorescence analyses (IgG, IgA, IgM, C3, C1q and C4) were all negative. These biopsy findings supported a diagnosis of FSGS.

After 3 years of treatment with an angiotensin receptor blocker (ARB), her proteinuria decreased. However, her serum creatinine level was $1.07 \mathrm{mg} / \mathrm{dL}$, and she still had stage 2 CKD.

\section{Discussion}

In Japan, birth weights have decreased over the years, which has resulted in decreased renal function in some healthy Japanese adolescents [8]. Birth weight correlates linearly with the total number of functional nephrons. In individuals born with a LBW, a congenital deficit of nephrons results in compensatory glomerular hyperfiltration, hypertrophy, and hypertension. The degree of susceptibility to the development of CKD is known to have a sex difference [9], and it has been demonstrated that renal function decreases more slowly in women than in men, and the effect of LBW on CKD is weaker in women [10]. Other than sex, several factors affect the development of CKD, such as race, obesity, hypertension, etc. Das et al. demonstrated that at least one-third of people with diabetes are born with a LBW and develop, and hence this risk is of great importance [11].

Even if an individual develops proteinuria, there may not be any abnormalities evident on renal biopsy. Hayashi et al. reported that 5 children born with very LBWs were diagnosed as having proteinuria, but their renal biopsies showed no findings of mesangial proliferation or glomerular sclerosis, although the mean diameter of their glomeruli was larger than that of normal glomeruli [12]. A second-hit exposure might be associated with the development of CKD. In the present case, the first hit might be the LBW, and the second-hit exposure might be hyperuricemia. Plasma uric acid levels are increased because of a decrease in glomerular filtration rate. The patient continued to show an increased serum creatinine level, and might hence require urate-lowering therapies in the future. Clinical studies have demonstrated that urate-lowering therapies might help to prevent and delay the worsening of CKD [13].

ARBs reduce glomerular hypertension, and have been reported to decrease proteinuria. In our present patient, her proteinuria decreased after 3 years of treatment with ARB. The birth history of patients is not usually confirmed by nephrologists. Obtaining information regarding the preterm birth and LBW of patients is important in the diagnosis 
of noncommunicable diseases, because urinary screening is not routinely performed in schools in countries other than Japan.

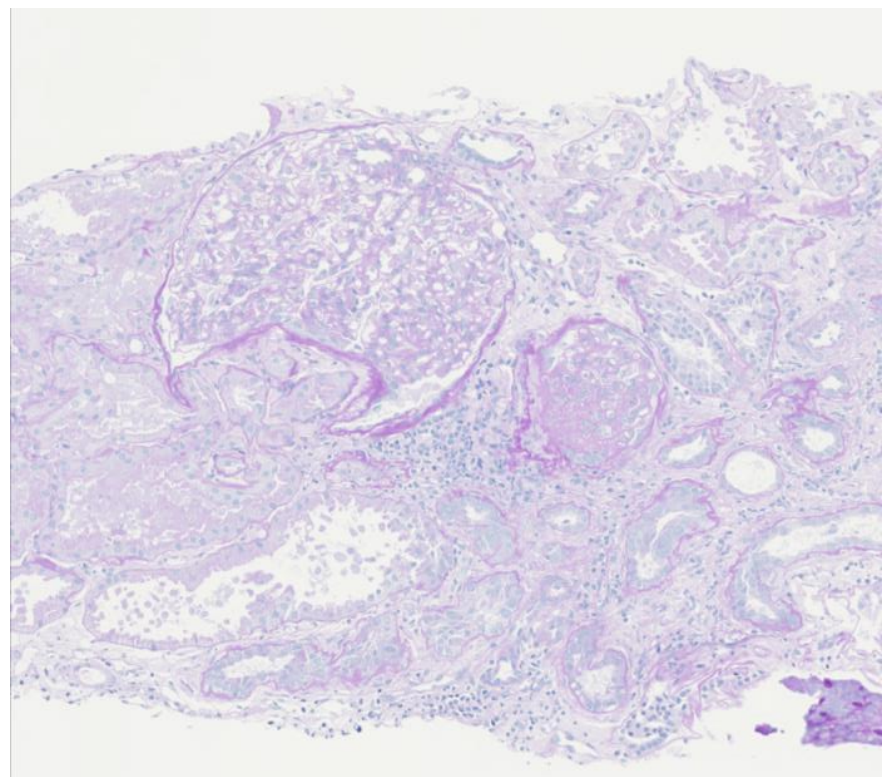

Figure 1. The mean diameter of the glomeruli of this patient $(348.23 \mu \mathrm{m})$ was much larger than that of normal glomeruli $(168 \pm 12 \mu \mathrm{m})$. Periodic Acid Schiff staining (magnification: $200 \times$ ).

Author Contributions: Y.K. wrote the manuscript; Y.K., K.A., and G.Y contributed to the conception and design of the manuscript; H.K. critically reviewed the manuscript and supervised the whole study process. All authors read and approved the final manuscript.

Funding: This research received no external funding.

Institutional Review Board Statement: Not applicable

Informed Consent Statement: Written informed consent has been obtained from the patient to publish this paper.

Data Availability Statement: Not applicable

Conflicts of Interest: The authors declare no conflicts of interest associated with this study.

\section{References}

[1] Blencowe, H.; Cousens, S.; Oestergaard, M.Z.; Chou, D.; Moller, A-B.; Narwal, R.; Adler, A.; Garcia, C.V.; Rohde, S.; Say, L.; Lawn, J.E. National, regional, and worldwide estimates of preterm birth rates in the year 2010 with time trends since 1990 for selected countries: a systematic analysis and implications. Lancet. 2012, 379, $2162-217$.

[2] Chehade, H.; Simeoni, U.; Guignard, J.P.; Boubred, F. Preterm Birth: Long Term Cardiovascular and Renal Consequences. Curr Pediatr Rev. 2018, 14, 219-226.

[3] Hanson, M.; Gluckman, P. Developmental origins of noncommunicable disease: Population and public health implications. Am. J. Clin. Nutr. 2011, 94, 1754S-1758S.

[4] Takemoto, Y.; Ota, E.; Yoneoka, D.; Mori, R.; Takeda, S. Japanese secular trends in birthweight and the prevalence of low birthweight infants during the last three decades: A population-based study. Sci Rep. 2016, 6, 31396.

[5] Hodgin, J.B.; Rasoulpour, M.; Markowitz, G.S.; D'Agati, V.D. Very low birth weight is a risk factor for secondary focal segmental glomerulosclerosis

Clin J Am Soc Nephrol. 2009, 4, 71-76.

[6] Clifford, S.M., Bunker, A.M., Jacobsen, J.R., Roberts, W.L. Age and gender specific pediatric reference intervals for aldolase, amylase, ceruloplasmin, creatine kinase, pancreatic amylase, prealbumin, and uric acid. Clin Chim Acta. 2011, 412, 788-790.

[7] Kambham, N.; Markowitz, G.S.; Valeri, A.M.; Lin, J.; D'Agati, V.D. Obesity-related glomerulopathy: an emerging epidemic. Kidney Int. 2001, 59, 1498-1509. 
[8] Murai-Takeda, A.; Kanda, T.; Azegami, T.; Hirose, H.; Inokuchi, M.; Tokuyama, H.; Wakino, S.; Tokumura, M.; Kawabe, H.; Mori, M.; Itoh, H. Low birth weight is associated with decline in renal function in Japanese male and female adolescents. Clin Exp Nephrol. 2019, 23, 1364-1372.

[9] Carrero, J.J. Gender differences in chronic kidney disease: underpinnings and therapeutic implications. Kidney Blood Press Res. 2010, 33, 383-392.

[10] Eriksson, J.G.; Salonen, M.K.; Kajantie, E.; Osmond, C. Prenatal Growth and CKD in Older Adults: Longitudinal Findings from the Helsinki Birth Cohort Study, 1924-1944. Am J Kidney Dis. 2018, 71, 20-26.

[11] Das, S.K.; Mannan, M.; Faruque, A.S.; Ahmed, T.; McIntyre, H.D.; Al, Mamun.A. Effect of birth weight on adulthood renal function: A bias- adjusted meta-analytic approach. Nephrology. 2016, 21, 547-565.

[12] Hayashi, A.; Santo, Y.; Satomura, K. Proteinuria and glomerular hypertrophy in extremely low-birthweight children. Pediatr Int. 2014, 56, 860-864.

[13] Kielstein, J.T.; Pontremoli, R.; Burnier, M. Management of Hyperuricemia in Patients with Chronic Kidney Disease: a Focus on Renal Protection. Curr Hypertens Rep. 2020, 22, 102. 